\title{
A Primary Capicua Transcriptional Repressor (CIC)- Rearranged Round Cell Sarcoma of the Neck: A Case Report and Literature Review
}

\author{
J bali $\mathbf{S}^{1 *}$, Ksontini FL ${ }^{2}$, Dhambri S', Chelly $\mathbf{B}^{3}$, \\ Mezlini $\mathbf{A}^{\mathbf{2}}$ and Gritli $\mathrm{S}^{1}$ \\ ${ }^{1}$ ENT Department, Salah Azaiez Institute of Cancer, \\ Medicine University, Tunis, Tunisia \\ ${ }^{2}$ Medical Oncology Department, Salah Azaiez Institute of \\ Cancer, Medicine University, Tunis, Tunisia \\ ${ }^{3}$ Department of Histopathology, La Rabta hospital, \\ Medicine University, Tunis, Tunisia \\ *Corresponding author: Souheil J bali, ENT \\ Department, Salah Azaiez Institute of Cancer, Medicine \\ University, Tunis, Tunisia
}

Received: August 09, 2021; Accepted: August 30, 2021; Published: September 06, 2021

\begin{abstract}
This is a case report of a CIC-rearranged sarcoma located in the neck. Our patient was a 36 year-old-woman with unremarkable past medical history who presented with a left lateralized neck mass evolving for one year without other signs. The physical examination showed a bulky left cervical mass of $12 \mathrm{~cm}$ long axis, with infected and necrotic areas. There was no palpable lymph nodes. A computed tomography (CT) scan was performed and confirmed the suspicious nature of the lesion and did not show distant metastasis. Histology (completed with Fluorescence in situ Hybridization (FISH) analysis) of a biopsy confirmed the diagnosis of round cell sarcoma with $\mathrm{ClC}$ rearrangement. As the tumor was unresectable, we started with chemotherapy but the tumor progressed after three cycles. The patient could not receive a second line chemotherapy because of the deterioration of the general condition and died after 1 month.
\end{abstract}

In conclusion, CIC-rearranged sarcoma is a rare tumor and has a poorer prognosis than the classic Ewing Sarcoma (ES). Its treatment is still challenging. More research is needed to establish the optimal treatment strategies.

Keywords: Round cell sarcoma; CIC rearrangement; Chemotherapy; Prognosis

\section{Introduction}

CIC-rearranged sarcomas have been relatively recently described as aggressive tumors arising in soft tissues of children and young adults. Although they share partial morphologic overlap with Ewing sarcoma (ES) and variable CD99 expression, emerging molecular data suggest that CIC-DUX4 tumors have a distinct pathogenesis [1]. This tumor presents as a round cell sarcoma, which is a heterogeneous group of undifferentiated sarcomas. In the recent years, identification of novel molecular alterations has greatly improved the classification. In addition to Ewing sarcoma, we currently recognize four main categories: CIC-rearranged sarcomas, BCOR-rearranged sarcomas, round cell sarcomas with EWSR1 gene fusion with non-ETS family members and the unclassified undifferentiated small round cell sarcoma [2]. The aim of this work is to describe a case of this rare tumor occurring in the neck with a review synthesis of the recent literature data.

\section{Case Presentation}

Our patient was a 36-year-old woman who presented with of a left lateral cervical mass evolving for one year (Figure 1a). Her history of disease goes back to one year marked by the appearance of a swelling of the left side of the neck, which gradually increased in size becoming painful. At the onset of the disease, there was no deterioration of the general state nor asthenia or weight loss. She had multiple medical consultations and multiple courses of antibiotics but the swelling continued increasing in size. She had no particular past medical history, family history and social history.
The physical examination showed a patient with a good performance status, a bulky left cervical mass of $12 \mathrm{~cm}$ long axis, multi-lobulated with presence of ulcerations, necrotic areas and signs of infection. There was no palpable lymph nodes in the physical examination. A cervical Computed Tomography (CT) scan was performed and showed huge soft tissue mass of the left posterior cervical space measuring $79 \times 67 \mathrm{~mm}$ in its major axes with multiples cervical and mediastinal lymph nodes (Figure 1). A biopsy was performed. It showed an undifferentiated neoplasm composed of small round tumor cells with round, open chromatic nuclei, and scant cytoplasm in a sheet growth pattern. Areas of geographic coagulative tumor cell necrosis were also noted. Immunohistochemically, ETV4 was diffusely positive. Immunostainings for CD99, KL1, CD34, S100,

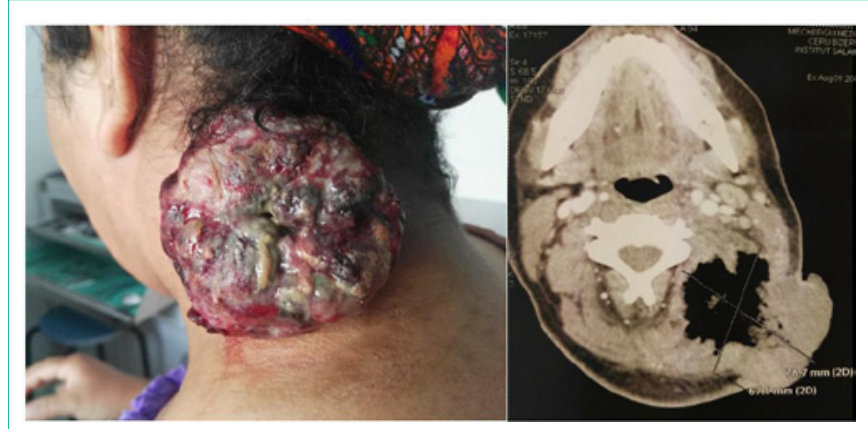

Figure 1: Clinical and imaging findings. 1a) (on the left): Clinical presentation: large, widely necrotic latero-cervical tumor. 1b) (on the right): CT scan (axial section): huge soft tissue masse of the left posterior cervical space measuring $79 \times 67 \mathrm{~mm}$ in its major axes.
Austin J Med Oncol - Volume 8 Issue 2 - 2021

ISSN : 2471-027X | www.austinpublishing group.com

Jbali et al. (C) All rights are reserved
Citation: Jbali S, Ksontini FL, Dhambri S, Chelly B, Mezlini A and Gritli S. A Primary Capicua Transcriptional Repressor (CIC)-Rearranged Round Cell Sarcoma of the Neck: A Case Report and Literature Review. Austin J Med Oncol. 2021; 8(2): 1066. 
Table 1: Molecular alteration in undifferentiated sarcomas with round cell phenotype.

\begin{tabular}{|c|c|c|c|}
\hline Histological Type & References & Molecular Alteration & Gene Fusion \\
\hline \multirow{6}{*}{ CIC sarcoma } & \multirow{2}{*}{ Italiano A and al (2012) [3] } & t (4;19)(q35;q13) & CIC-DUX4 \\
\hline & & $\mathrm{t}(10 ; 19)(\mathrm{q} 26 ; \mathrm{q13})$ & CIC-DUX4 \\
\hline & \multirow{2}{*}{ Sugita and al (2014) [4] } & \multirow{2}{*}{$\mathrm{t}(\mathrm{x} ; 19)(\mathrm{q13} ; \mathrm{q13.3})$} & CIC-FOXO 4 \\
\hline & & & CIC-LEUTX \\
\hline & \multirow{2}{*}{ Le Loarer F and al (2017) [2] } & t (15;19)(q14 ;q13.2) & CIC-NUTM1 \\
\hline & & t $(10 ; 19)(q 23.3 ; q 13)$ & CIC-NUTM2 \\
\hline \multirow{3}{*}{ BCOR sarcoma } & Pierron G (2012) [5] & $\operatorname{Inv}(X)(p 11 ; p 11)$ & BCOR-CCNB3 \\
\hline & Specht and al (2016) [6] & $t(4 ; x)(p 11, q 31)$ & BCOR-MAML3 \\
\hline & Specht and al (2016) [6] & $t(X ; 22)(p 11, q 13.2)$ & BCOR-ZC3H7B \\
\hline \multirow{2}{*}{ EWSR1-NonETS sarcoma } & Sadri N and al (2014) [7] & t (20;22)(q13.2;q12) & EWSR1-NFATC2 \\
\hline & Mastrangelo and al (2000) [9] & $\operatorname{lnv}(22)(q 12 ; q 12)$ & EWSR1-PATZ 1 \\
\hline
\end{tabular}

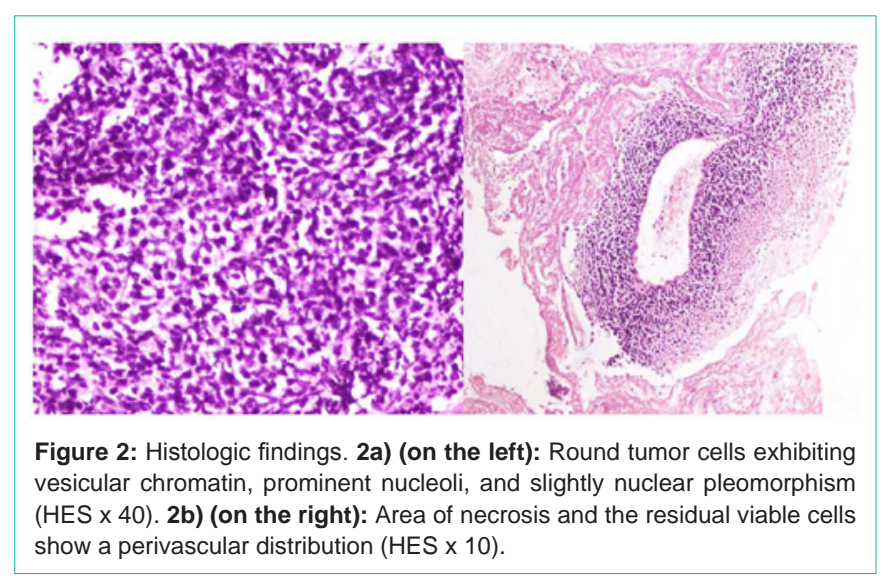

Desmin, MDM2, Caldesmon and cyclin B3 were absents. Therefore, we concluded to an undifferentiated round cell sarcoma (Figure 2). A FISH study showed a rearrangement of the CIC gene.

A thoraco-abdomino-pelvic CT scan was carried and was normal. After multidisciplinary meeting, we decided to start with chemotherapy because the lesion was not resectable. The patient received soft tissue sarcoma regimen (three cycles of doxorubicin and ifosfamide) with moderate tolerance but a clinical progression. Radiological evaluation showed also a metastatic progression to the lung. A second line chemotherapy was proposed but the patient came with a deterioration of the general condition and we indicated supportive care. She died after 1 month.

\section{Discussion}

This is one of the reports of a CIC rearranged round cell sarcoma diagnosed by molecular genetic research. This entity is the second most frequent type of round cell sarcomas that morphologically resemble to classical ES but is molecularly different.

Until now, small round cell sarcomas look, histologically, like classical Ewing sarcoma but without the EWSR-ETS fusion gene or translocation.

They show small to medium size cells with a solid pattern of growth and no stroma. However, for a few years, increased use of modern molecular methods based on next-generation sequencing have enabled the identification of distinct subgroups based on the discovery of novel molecular driving events. We currently recognize four main categories: CIC-rearranged sarcomas, BCOR-rearranged sarcomas, round cell sarcomas with EWSR1 gene fusion with nonETS family members and the unclassified undifferentiated small round cell sarcomas.

Known molecular alterations in undifferentiated sarcomas with round cell phenotype are summarized in Table 1.

CIC rearranged sarcoma is the most frequent and bestcharacterized subgroup of the family of undifferentiated sarcomas with round cell phenotype. CIC-rearranged sarcoma was first reported in 2006 as Ewing-like sarcoma by Kawamura-Saito. This tumor represents an undifferentiated round cell malignancy, characterized by recurrent CIC (capicua transcriptional repressor) gene rearrangements [3]. The CIC gene is the human homolog of the Drosophila gene Capicua that encodes a high-mobility group box transcription factor and is involved in the development of the central nervous system. The CIC gene present on chromosome 19 acts as a strong transcriptional activator by fusion with the DUX4 gene present on chromosome 4 or 10 , causing a t $(4 ; 19)$ or $t(10 ; 19)$ translocation. DUX4 is the most common fusion partner [3]. A fusion with nonDUX4 gene partners (FOXO4, LEUTX, NUTM1, and NUTM2A) occurs in approximately $5 \%$ of cases [4].

This tumor affects young adults with a peak incidence in the fourth decade, with slight male predominance, but it can occur at any age [3]. Patients are generally older than in ES. About $90 \%$ of cases arise in soft tissues. It can occur equally in the extremities, trunk/pelvis, head and neck. It arises in visceral organs in approximately $10 \%$ of cases [10]. Whereas superficial soft tissues can be involved in less than $10 \%$ of cases. In the study of Antonescu CR et al., authors investigates the clinico-pathologic features of a large cohort of sarcomas with CIC gene rearrangement (115 patients) [11]. The mean age was 32 years. Most tumors occurred in the soft tissue (86\%), predominantly deepseated and equally divided between trunk and extremity, followed by visceral locations (12\%) and rarely in the bone (3\%).

According to the series published recently by Le Loarer et al, the CIC-NUTM1 variant represent a new molecular variant of CIC- 
focused sarcomas with a predilection for the central nervous system [2].

There are certain distinctive features associated with CICDUX4 positive tumors: the most important is a wider spectrum of cytomorphology with mixture of round, spindle and epithelioid cells. Tumor cells also show increased nuclear size and shape variability, vesicular chromatin with focally prominent nucleoli, in addition to more abundant (typically light eosinophilic) cytoplasm [11]. Mitotic count is generally high (often exceeding 40 mitoses/10 HPF). Neoplastic cells are often organized in a lobular growth pattern, associated with presence of fibrous septa. Confluent geographic areas of necrosis represent a relatively frequent finding [12].

Stromal myxoïd change is a common finding in CIC-positive tumors, which is typically absent in Ewing sarcoma [11]. Most CICrearranged tumors (84\%) shows variable expression of CD99, but only $23 \%$ with a diffuse pattern and $16 \%$ are completely negative. Nuclear WT1 reactivity is also a consistent finding in CIC-positive sarcomas, in contrast with Ewing Sarcoma. Nuclear expression of DUX4 is consistently present. ETV4 is diffusely expressed as a consequence of the genomic up regulation of the ETV4 gene; however, it is not entirely specific [12].

Regarding treatment, the best strategy for these patients remains unclear. It is believed that these tumors have a more aggressive course when compared with classic Ewing Sarcoma. Complete surgery seems to be the most effective treatment for the CIC-rearranged sarcoma.

However, the effectiveness of chemotherapy is still debated. CICrearranged sarcoma is resistant to chemotherapy and often follows a more aggressive clinical course. Most of available data about chemotherapy are limited to retrospective case reports or series. In most studies with neoadjuvant chemotherapy, the regimes used were vincristine, Ifosfamide, doxorubicin and Etoposide. Responses were in fact minimal, and if achieved, were generally transient with rapid progression owing to drug resistance. Therefore, clinicians must carefully consider the approach in patients who present with localized disease and may be treat them similarly to soft tissues sarcomas. The best strategy is not clear yet and prospective collection of multiinstitutional data will help in evaluating the response to neo-adjuvant chemotherapy. Even in our patient, the tumor had progressed and the patient developed early local recurrence and distant metastases to the lung and liver and died despite first and second line chemotherapies [13].

In our study, the prognostic of our patient was very poor with a short survival. These findings are confirmed by the results of the cohort of Antonescu CR et al. [11]. IN this study the 5-year, overall survival was $43 \%$ for the entire group and $49 \%$ for the patients who presented with localized disease at diagnosis. The overall survival was significantly lower compared to the localized Ewing sarcoma cohort, matched for stage and age, which showed a 5 year-survival of $76 \%$. Yoshida et al found, also, a statistically significant inferior overall survival in a smaller cohort of 20 CIC-rearranged sarcomas compared to a group of 53 Ewing sarcoma patients [14].
The prognosis of CIC-rearranged sarcoma remains poor with a high metastatic risk. Most metastases sites reported were the lung, the liver, brain, pleura, thyroid, bone, and soft tissues.

\section{Conclusion}

We report a case of highly aggressive CIC-rearranged sarcoma of the neck with poor outcome and very short survival. These sarcomas are highly malignant with a poor prognosis even after radical resection. The accumulation of further cases is desirable to establish optimal treatment strategies.

\section{References}

1. Yoshimoto T, Tanaka M, Homme M, Yamazaki Y, Takazawa Y, Antonescu $\mathrm{CR}$, et al. CIC-DUX4 Induces Small Round Cell Sarcomas Distinct from Ewing Sarcoma. Cancer Res. 2017; 77: 2927-2937.

2. Le Loarer F, Pissaloux D, Coindre JM, Tirode F, Vince DRJSPC. Update on families of round cell sarcomas other than classical Ewing sarcomas. 2017; 10: $587-620$

3. Italiano A, Sung YS, Zhang L, Singer S, Maki RG, Coindre JM, et al. High prevalence of CIC fusion with double-homeobox (DUX4) transcription factors in EWSR1-negative undifferentiated small blue round cell sarcomas. 2012; 51: 207-218.

4. Sugita S, Arai Y, Tonooka A, Hama N, Totoki Y, Fujii T, et al. A novel ClCFOXO4 gene fusion in undifferentiated small round cell sarcoma: a genetically distinct variant of Ewing-like sarcoma. 2014; 38: 1571-1576.

5. Pierron G, Tirode F, Lucchesi C, Reynaud S, Ballet S, Cohen-Gogo S, et al. A new subtype of bone sarcoma defined by BCOR-CCNB3 gene fusion. 2012; 44: 461-466.

6. Specht K, Zhang L, Sung Y-S, Nucci M, Dry S, Vaiyapuri S, et al. Novel BCOR-MAML3 and ZC3H7B-BCOR gene fusions in undifferentiated small blue round cell sarcomas. 2016; 40: 433

7. Sadri N, Barroeta J, Pack SD, Abdullaev Z, Chatterjee B, Puthiyaveettil R, et al. Malignant round cell tumor of bone with EWSR1-NFATC2 gene fusion. 2014; 465: 233-239.

8. Sumegi J, Nishio J, Nelson M, Frayer RW, Perry D, Bridge JAJMP. A novel $\mathrm{t}(4 ; 22)(\mathrm{q} 31 ; \mathrm{q} 12)$ produces an EWSR1-SMARCA5 fusion in extra skeletal Ewing sarcoma/primitive neuroectodermal tumor. 2011; 24: 333-342.

9. Mastrangelo T, Modena P, Tornielli S, Bullrich F, Testi MA, Mezzelani A, et al. A novel zinc finger gene is fused to EWS in small round cell tumor. Oncogene. 2000; 19: 3799-3804.

10. Aoki Y, Oshiro H, Yoshida A, Morishima K, Miki A, Sasanuma H, et al. Pancreaticoduodenectomy for a primary duodenal capicua transcriptional repressor $(\mathrm{CIC})$-rearranged sarcoma with severe bleeding: a case report. BMC Gastroenterol. 2020; 20: 1-6

11. Antonescu CR, Owosho AA, Zhang L, Chen S, Deniz K, Huryn JM, et al. Sarcomas with $\mathrm{CIC}$-rearrangements are a distinct pathologic entity with aggressive outcome: a clinicopathologic and molecular study of 115 cases. Am J Surg Pathol. 2017; 41: 941.

12. Sbaraglia M, Righi A, Gambarotti M, Dei Tos APJVA. Ewing sarcoma and Ewing-like tumors. Virchows Arch. 2020: 1-11.

13. Renzi S, Anderson ND, Light N, Gupta A. Ewing-like sarcoma: An emerging family of round cell sarcomas. J Cell Physiol. 2019; 234: 7999-8007.

14. Yoshida A, Goto K, Kodaira M, Kobayashi E, Kawamoto H, Mori T, et al. CICrearranged Sarcomas: A Study of 20 Cases and Comparisons With Ewing Sarcomas. Am J Surg Pathol. 2016; 40: 313-323. 